\title{
Kink solutions of the binormal flow
}

\author{
Luis Vega
}

\section{Abstract}

In these pages we shall present some recent work done in collaboration with S. Gutiérrez on the selfsimilar solutions of the binormal flow

$$
X_{t}=X_{s} \wedge X_{s s}
$$

Here

$$
X(s, t) \in \mathbf{R}^{3}, \quad s \in \mathbf{R}, t \in \mathbf{R}
$$

and $s$ represents the arclength parameter

$$
|\dot{X}(s, t)|^{2}=1 .
$$

Differentiation in (1) gives

$$
X_{s t}=X_{s} \wedge X_{s s s}
$$

and as long as $X$ is regular enough we get

$$
\frac{d}{d s}\left|X_{s}\right|^{2}=X_{s}\left(X_{s}\right)_{t}=X_{s} \cdot\left(X_{s} \wedge X_{s s s}\right)=0 .
$$

This flow was found for the first time by Da Rios [DaR] in 1906 in his Tesis di Laurea under the direction of Levi-Civita as an approximation of the motion of a vortex tube of infinitesimal cross section (vortex filament) within an inviscid ideal fluid.

This amounts to say that the vorticity $\omega=\nabla \times u$, with $u$ the velocity of the fluid, is a singular vectorial measure with support on a curve in $\mathbf{R}^{3}$ (precisely $X(s, t)$ ). The vectorial identity

$$
\nabla \times(\nabla \times u)=-\Delta u+\nabla \operatorname{div} u=-\Delta u
$$

allows us to compute the velocity field $u$ from the vorticity $w$ by the Biot-Savart integral

$$
u(P)=-\frac{\Gamma}{4 \pi} \int_{-\infty}^{\infty} \frac{P-X(s)}{|P-X(s)|^{3}} \times T(s) d s
$$


with $X_{s}(s)=T(s)$ and $\Gamma$ the circulation of the vortex.

In the above integral we have assumed that the filament has infinite length and goes to infinity when $s$ goes to $\pm \infty$. In order to find the law for the evolution of the filament one identifies the velocity of a point $Q$ in the filament with that one of the fluid at that point. However, the integral is highly singular and some summation method is needed to compute the limit when $P$ approaches $Q$. Da Rios considers a Taylor expansion of $X(s, t)$ at the point $Q$ and writes it with the use of the Frenet frame. The first term is the usual angular velocity and which grows like the inverse of the distance to the filament and that also appears in the case of a straight filament. The overall effect of this rotating component of the velocity is null with respect to the displacement of the filament. The second term is proportional to the curvature and in the direction of the binormal. It is precisely this one that is captured by (1), which in the Frenet system is written as

$$
X_{t}=c b
$$

with $c$ the curvature and $\mathrm{b}$ the binormal.

This approximation involves some logarithmic divergence proportional to the portion of filament which is considered to evaluate the Biot-Savart integral (2). When a slender vortex tube is taken instead of a vortex filament the logarithmic divergence is proportional also to the core of the tube. See for example [Sa] for the deduction of (3) as well as for an analysis of the limitations of this model. Notice that this approximation only takes into consideration the parts of filament close to the given point Q. For this reason the binormal flow also is known as the Local Induction Approximation (LIA) in the context of vortex dynamics. Also observe that this model preserves three fundamental properties of Euler equation. The first one is that vortex tubes travel with the fluid (in our setting this is given by the fact we are considering flow of curves, each curve representing a vortex tube of infinitesimal cross section). The second one is that the circulation along any closed curve $\gamma$ on the tube is constant. This latter property is a consequence of Stokes theorem. So that the circulation is equal to the surface integral of the vorticity on the section of the tube which has $\gamma$ as its boundary. In the ideal situation of a filament this section is shrinked to the corresponding point of the tube. Then the vorticity is identified by a delta-vector measure in the direction of the tangent vector $T$ at the given point $Q$ and with modulus $|\Gamma|$. From this point of view it is natural to assume that the flow preserves the arc-length parametrization. There is a high prize to pay for this, which is that if for example we start with a closed filament the total length will be preserved and this is highly unexpected when a vortex tube of positive cross section is considered. And finally the binormal flow is reversible in time.

The solutions of (1) we will consider in these pages are examples of kink singularties which are developped in finite time starting whith analytic curves. In the setting of Euler equations it is known to be necessary a strong streching of the vortex tube for such singularities to occur. Let us recall at this respect the result of Constantin,Feffermann and Majda [CFM]. Under some extra hypothesis, these authors give as a necessary condition for the development of a singularity at time 
$T^{*}$ that

$$
\int_{0}^{T^{*}} \sup _{x}\|\nabla \zeta\|_{L^{\infty}}^{2} d t=\infty .
$$

where $\zeta=\frac{\omega}{|\omega|}$ is the direction of the vorticity vector. In our setting the rôle of $\zeta$ is played by the tangent vector $T$ and the analogous statement to (4) should be

$$
\int_{0}^{T^{*}} \sup _{s}\left|T_{s}\right|^{2} d t=\int_{0}^{T^{*}} \sup _{s}|c|^{2} d t=\infty .
$$

We shall see that (5) is satisfied by our solutions.

Another aspect to take into account when comparing (2) with Euler equations is the conservation laws preserved by the binormal flow, see [DaR]. We have the following ones

i) $\int c^{2} d s$, kinetic energy,

ii) $\frac{1}{2} \int X \wedge \omega$, linear momentum,

iii) $\frac{1}{3} \int X \wedge(X \wedge \omega)$, angular momentum,

iv) $\int c^{2} \tau$, helicity,

v) $\int \tau$, total torsion.

In fact the binormal flow is a completely integrable system and has an infinite number of conservation laws.

\section{Symmetries}

The flow is invariant under rotations, translations in space and time, and under one parameter family of scalings:

If $\lambda>0$ and $\lambda^{\alpha} X\left(\lambda s, \lambda^{\beta} t\right)=X_{\lambda}$ is a solution of (1) so is $X$ if

$$
-\alpha+\beta=3 .
$$

Of these scalings the only one that preserves arclength is $\alpha=-1, \beta=2$. If we consider $T$ instead of $X$ we get $T_{\lambda}(s)=T\left(\lambda s, \lambda^{2} t\right)$. This scaling is also the one inherited by the vorticity vector $\zeta$ in the zero viscosity limit of Navier-Stokes equation.

The binormal flow has some other unexpected symmetries. Consider the pair $(c, \tau)$ given by the curvature and torsion of $X$. It is easy to obtain (Da Rios and Betchov equations [DaR] and [Be]) the system of equations of $(c, \tau)$ if $X$ evolves according to the binormal flow. It is the following:

$$
\begin{aligned}
c_{t} & =-(\tau c)_{s}-c_{s} \tau \\
\tau_{t} & =\left(\frac{c_{s s}-c \tau^{2}}{c}\right)_{s}+c_{s} c .
\end{aligned}
$$


Given $N \in \mathbf{R}$ define

$$
\left(c_{N}, \tau_{N}\right)=(c(s-2 t N), \tau(s-2 t N)+N) .
$$

Then if $(c, \tau)$ solves $(6)$, so does $\left(c_{N}, \tau_{N}\right)$. This symmetry at time zero amounts to just an increase by $N$ of the torsion

$$
\left(c_{N}(0), \tau_{N}(0)\right)=(c(0), \tau(0)+N) .
$$

As we have already said the binormal flow is time reversible: if $X$ is a solution, $\tilde{X}(s, t)=-X(s,-t)$ is also a solution. A final relevant fact about (1) is a remarkable transformation due to Hasimoto [Ha]. It is as follows. Define the filament function

$$
\Psi(s, t)=c(s, t) \exp \left(\int_{0}^{s} \tau\left(s^{\prime}, t\right) d s^{\prime}\right) .
$$

Then if $X$ solves (1), $\Psi$ is a solution of the cubic nonlinear Schrödinger equation

$$
i \Psi_{t}+\Psi_{s s}+\frac{1}{2}\left(|\Psi|^{2}+A(t)\right) \Psi=0 .
$$

\section{Selfsimilarity : Kink solutions}

We will focus our attention in the scaling $\alpha=-1, \beta=2$. Assume

$$
X_{\lambda}=X \quad \forall \lambda>0
$$

Differentiating w.r.t. $\lambda$ and taking $\lambda=1$ we get

$$
-X+s X_{s}+2 X_{t}=0 .
$$

If $X_{t}$ solves (1) and calling $G(s)=X(s, 1)$, we get the ODE

$$
-G+s G^{\prime}-2 G^{\prime} \times G^{\prime \prime}=0 .
$$

If $\mathrm{G}$ solves (9) then

$$
X(s, t)=\sqrt{t} G\left(\frac{s}{\sqrt{t}}\right)
$$

is a solution of (1). Differentiating (9) and calling (with abuse of notation) $T=G^{\prime}$, we get

$$
s T^{\prime}=2 T \times T^{\prime \prime}
$$

From the Frenet system

$$
s c n=s T^{\prime}=2 T \times\left(c^{\prime} n+c n^{\prime}\right)=2 c^{\prime} b+2 c \tau n .
$$

Hence, see [LRT] and [Bu],

$$
\begin{aligned}
& c=c_{0} \\
& \tau=s / 2 .
\end{aligned}
$$


It is a remarkable fact that if instead of assuming scaling invariance we consider the symmetry given by (7), we would have arrived at the same conditions (11). - See [GRV].

In [GRV] a careful analysis of the curves given by (11) is made. In particular the following theorem is proved.

Theorem 1 ([GRV]) Given $\left(A^{+}, A^{-}\right) \in \mathcal{S}^{2} \times \mathcal{S}^{2}$ with $A^{+}+A^{-} \neq 0$, there is a unique $c_{0}$ such that for $X(s, t)=\sqrt{t} G_{C_{0}}(s / \sqrt{t})$

(i) $\left|X(s, t)-s\left(A^{+} \chi_{[0,+\infty)}(s)+A^{-} \chi_{(-\infty, 0]}(s)\right)\right| \leq c_{0} \sqrt{t}$ as $s>>1$,

(ii) $G(s)=A^{ \pm}\left(s+2 \frac{c_{0}^{2}}{s}\right)-4 c_{0} \frac{n(s)}{s^{2}}+O\left(\frac{1}{s^{3}}\right)$ as $s \rightarrow \pm \infty$,

(iii) $T(s)=A^{ \pm}-2 \frac{c_{0}}{s} b(s)+O\left(\frac{1}{s^{2}}\right)$ as $s \rightarrow \pm \infty$,

(iv) $n-i b=B^{ \pm}\left(c_{0}\right) e^{i\left(s^{2} / 4+c_{0}^{2} \log s / 2\right)}+O\left(\frac{1}{s}\right)$ as $s \rightarrow \pm \infty$, and $B^{ \pm}\left(c_{0}\right) \cdot A^{ \pm}=0$, for some $B^{ \pm}$,

(v) $\cos \left(\frac{\left(A^{+}, A^{-}\right)}{2}\right)=e^{-c_{0}^{2} / 2 \pi}$,

(vi) There are $\tilde{c_{0}}, \tilde{\tilde{c_{0}}}$ such that if $c_{0}<\tilde{c_{0}}$ there are no selfintersections and if $c_{0}>\tilde{\tilde{c_{0}}}$ there are a finite number of them.

\section{Remarks}

In terms of nonlinear Schrödinger equation this theorem says nothing. In fact, from (11) we immediately compute that given $c_{0}$, the filament function is

$$
\psi_{c_{0}}=\frac{c_{0}}{\sqrt{t}} e^{s^{2} / 4 t}
$$

We recover condition (5)

$$
\int_{0}^{1} \sup _{s}\left|T_{s}\right|^{2} d t=\int_{0}^{1} \frac{c_{0}^{2}}{t} d t=\infty .
$$

The scaling $\sqrt{t} G(s / \sqrt{t})$ gives for $\tilde{\Psi}$ the following one

$$
\begin{aligned}
& \tilde{\Psi}(s, t)=\frac{1}{\sqrt{t}} \Psi\left(\frac{s}{\sqrt{t}}\right), \\
& A(t)=\frac{\alpha}{t} \quad \alpha \in \mathbf{R}
\end{aligned}
$$

Hence one would expect a two parameter family of solutions of (8). In fact, a simple computation gives that if $\Psi$ solves the scalar ODE

$$
\begin{gathered}
\Psi^{\prime \prime}-\frac{i}{2}\left(\Psi+s \Psi^{\prime}\right)+\frac{\Psi}{2}\left(|\Psi|^{2}+\alpha\right)=0, \\
\Psi(0)=\Psi_{0}, \quad \Psi^{\prime}(0)=\Psi_{1} .
\end{gathered}
$$


A natural question is why we only obtain a one parameter family of curves $G$. A first answer is that when we introduce the invariance under rotation of the binormal flow we recover all the possible curves. Those come from the precise choice $\Psi(0)=c_{0}$, $\Psi^{\prime}(0)=0, \alpha=-c_{0}{ }^{2}$. But still remains the question of what happens with the other solutions of (12). Notice that the corresponding solutions to the above choice have as initial data for the evolution equation $(8) \tilde{\Psi}(s, 0)=c_{0} \delta$. Hence other choices of (12) should be related to $\tilde{\Psi}(s, 0)=c_{0}$ p.v. $\frac{1}{s}$. One integration of this gives

$$
\int^{s} \text { p.v. } \frac{1}{s}=\log |s|
$$

which breaks the scaling symmetry.

This remark suggests to look for almost selfsimilar solutions of the binormal flow (1). Given $\mathcal{A} \in \mathcal{M}_{3 \times 3}$ real and antisymmetric (hence $e^{\mathcal{A} / 2}$ is a rotation), we consider the following ansatz

$$
X(s, t)=e^{\mathcal{A} / 2 \log t} \sqrt{t} G(s / \sqrt{t}) .
$$

If we impose $X$ to be a solution of (1) we get that $G$ has to solve the vector ODE

$$
(\mathcal{I}+\mathcal{A}) G-s G^{\prime}=2 G^{\prime} \times G^{\prime \prime}, \quad\left|G^{\prime}(s)\right|^{2}=1 .
$$

Multiplication by $G^{\prime}$ and the vectorial identity $F \times(G \times H)=(F \cdot H) G-(F \cdot G) H$ give

$$
G^{\prime \prime}=\frac{1}{2}(\mathcal{I}+\mathcal{A}) G \times G^{\prime}, \quad(\mathcal{I}+\mathcal{A}) G(0) \cdot G^{\prime}(0)=0 .
$$

It is easy to prove that if $G$ satisfies (16) then is also a solution of (15). Given any initial condition $\left(G(0), G^{\prime}(0)\right)$ with $\left|G^{\prime}(0)\right|^{2}=1$ is very easy to prove global existence of $G$. In fact, we immediately obtain by multiplication by $G^{\prime}$ that

$$
\frac{d}{d s}\left|G^{\prime}(s)\right|^{2}=0
$$

Hence, from the local existence theory of ODE's, we conclude that

$$
\left(\begin{array}{c}
G \\
G^{\prime}
\end{array}\right)^{\prime}=\left(\begin{array}{c}
1 \\
\frac{1}{2}(\mathcal{I}+\mathcal{A}) G \times G^{\prime}
\end{array}\right)
$$

has a unique global solution for all $s$. Another bonus of solving (16) instead of (15) is that it can be analyzed numerically much easily -see for example the figures in my homepage.

Our next step is to look at the asympotitcs of the solutions of (16). To do so, it is better to consider

$$
F(s)=e^{-\mathcal{A} \log |s|} \frac{G(s)}{s} \quad s \neq 0 .
$$


Then

$$
\begin{aligned}
F^{\prime}(s) & =\left[\frac{1}{s}(-\mathcal{A}-\mathcal{I}) G+G^{\prime}\right] \frac{e^{-\mathcal{A} \log |s|}}{s} \\
& =-2 e^{-\mathcal{A} \log |s|} \frac{G^{\prime} \times G^{\prime \prime}}{s^{2}} \\
& =-2 e^{-\mathcal{A} \log |s|} \frac{c b}{s^{2}} .
\end{aligned}
$$

Assume $|c(s)|$ is bounded then

$$
\left|\int_{s}^{\infty} \frac{c\left(s^{\prime}\right) b\left(s^{\prime}\right)}{s^{\prime 2}} d s^{\prime}\right|<\infty, \quad\left|\int_{-\infty}^{s} \frac{c\left(s^{\prime}\right) b\left(s^{\prime}\right)}{s^{\prime 2}} d s^{\prime}\right|<\infty
$$

and from (18) there exists $A^{ \pm}$such that

$$
F(s)=A^{ \pm}+O(1 / s)
$$

Hence from (17)

$$
G(s)=s e^{-\mathcal{A} \log s} A^{ \pm}+O\left(\frac{1}{s}\right) .
$$

In order to complete the argument we need to prove that the curvature $c$ is bounded. It is here where NLS equation (8) plays a fundamental role. Following Hasimoto [Ha] we define the filament function as before

$$
\tilde{\Psi}(s, t)=c(s, t) e^{i \int_{0}^{s} \tau\left(s^{\prime}, t\right) d s^{\prime}} .
$$

The normal plane to the tangent vector is identified with the complex plane. Define the complex vector $N$

$$
N(s, t)=(n+i b)(s, t) e^{i \int_{0}^{s} \tau\left(s^{\prime}, t\right) d s^{\prime}} .
$$

Then simple computations give

$$
i \tilde{\Psi}_{t}+\tilde{\Psi}_{s s}+\frac{\tilde{\Psi}}{2}\left(|\tilde{\Psi}|^{2}+\alpha(t)\right)=0
$$

for some $\alpha(t)$.

Now assume the ansatz given in (14). Then

$$
\alpha(t)=-i\left(N_{t} \cdot \bar{N}\right)(0, t)-\frac{c_{0}^{2}}{t}=\frac{1}{t}\left((\mathcal{A} b \cdot n)(0)-c_{0}^{2}\right), \quad c_{0}=c(0,0)
$$

and $\tilde{\Psi}$ is selfsimilar

$$
\tilde{\Psi}(s, t)=\frac{1}{\sqrt{t}} \Psi(s / \sqrt{t}) .
$$

Hence $\Psi$ solves the complex ODE:

$$
\Psi^{\prime \prime}-\frac{i}{2}\left(\Psi+s \Psi^{\prime}\right)+\frac{\Psi}{2}\left(|\Psi|^{2}+\alpha\right)=0, \quad \alpha=\alpha(1) .
$$


Next we define $f$ by

$$
\Psi(s)=e^{i s^{2} / 4} f(s)
$$

to obtain

$$
f^{\prime \prime}+i \frac{s}{2} f^{\prime}+\frac{f}{2}\left(|f|^{2}+\alpha\right)=0 .
$$

After multiplication by $\bar{f}^{\prime}$ take the real part to obtain

$$
\frac{d}{d s}\left(\left|f^{\prime}\right|^{2}+\frac{1}{4}\left(|f|^{2}+\alpha\right)^{2}\right)=0
$$

So in particular $|f|^{2}+\alpha$ is bounded. And therefore $|f|^{2}=c^{2}$ is bounded as we wanted. Notice that, however, the above argument does not allow to conclude that $\left(A^{+}, A^{-}\right)$is not trivial. There is a simple way to prove that if $\mathcal{A}$ is sufficiently close to the identity matrix then necessarily $\left(A^{+}, A^{-}\right) \neq(0,0)$. This follows from (18), (19) and Theorem 1. Nevertheless we have a much stronger result.

Theorem 2 Given $\left(G(0), G^{\prime}(0)\right)$ with $\left|G^{\prime}(0)\right|^{2}=1$, there exist a unique $\left(A^{+}, A^{-}\right) \in$ $\mathbf{R}^{3} \times \mathbf{R}^{3},\left(a_{+}, a_{-}\right) \in[0,2 \pi) \times[0,2 \pi)$ and $\left(b^{+}, b^{-}\right) \in[0, \infty) \times[0, \infty)$ such that if $s \rightarrow \pm \infty$,

(i) $G(s)=s e^{\mathcal{A} \log |s|} A^{ \pm}+\frac{e^{\mathcal{A} \log |s|}}{s}\left(2 c_{ \pm \infty}{ }^{2} B^{ \pm}-a\left(e_{3} \times B^{ \pm}\right) \times B^{ \pm}+O\left(1 / s^{2}\right)\right)$,

(ii) $T(s)=e^{\mathcal{A l o g}|s|} B^{ \pm}-2 \frac{c_{ \pm \infty} b}{s}+O\left(1 / s^{2}\right)$,

(iii) $c_{ \pm \infty}(n-i b)(s)=b_{ \pm} e^{i\left(a_{ \pm}+\frac{s^{2}}{4}-\gamma_{ \pm} \log \left(\frac{|s|}{2}\right)-\frac{\pi}{2}\right)} e^{\mathcal{A} \log |s|}\left(\left(a\left(e_{3} \times B^{ \pm}\right) B^{ \pm}\right) \times B^{ \pm}-i a\left(e_{3} \times\right.\right.$ $\left.\left.B^{ \pm}\right)\right)+O(1 /|s|)$

where

$$
\begin{aligned}
B^{ \pm}=(\mathcal{I}+\mathcal{A}) A^{ \pm}, & \left|B^{ \pm}\right|=1, \quad \mathcal{A}=\left(\begin{array}{ccc}
0 & -a & 0 \\
a & 0 & 0 \\
0 & 0 & 0
\end{array}\right) \quad a \in \mathbf{R} \\
b_{ \pm}{ }^{2}= & \frac{c_{ \pm \infty}{ }^{2}}{4}\left(a^{2}-\left(c_{ \pm \infty}{ }^{2}+\alpha\right)^{2}\right) \\
& \gamma_{ \pm}=-3 c_{ \pm \infty}{ }^{2}-2 \alpha \\
& \alpha=\mathcal{A} b \cdot n(0)-c_{0}{ }^{2} .
\end{aligned}
$$

Reciprocally, we can fix the data at infinity and to be able to find a unique $\left(G(0), G^{\prime}(0)\right)$ such that the corresponding asymptotics at infinity are the given data. We have the following result.

Theorem 3 Given $B^{+}=\left(B_{1}^{+}, B_{2}^{+}, B_{3}^{+}\right) \in \mathcal{S}^{2}$ with $B_{3}^{+} \neq \pm 1, a_{+} \in[0,2 \pi)$ and $b_{+}>$ 0 , there exists a unique $\left(G(0), G^{\prime}(0)\right) \in \mathbf{R}^{3} \times \mathcal{S}^{2}$ such that the corresponding solution of (15) verifies $(i)$, (ii) and (iii) of Theorem 2 if $s \rightarrow+\infty$ and $B^{+}=(\mathcal{I}+\mathcal{A}) A^{+}$. $A$ similar result can be obtained at $-\infty$. 
The proof of Theorem 2 relies on a very delicate study of the asymptotics of the solutions to the complex ODE (25). It turns out that in order to integrate (25) is better to use the real variables.

$$
h=\operatorname{Im}\left(f \bar{f}^{\prime}\right) ; \quad y=\frac{d}{d s}|f|^{2}
$$

and to consider the system

$$
\begin{aligned}
& y^{\prime}(s)=s h+g\left(|f|^{2}\right), \\
h^{\prime}(s)=-\frac{s}{4} y &
\end{aligned}
$$

with

$$
\begin{gathered}
g\left(|f|^{2}\right)=2 E(0)-\frac{1}{2}\left(3|f|^{2}+\alpha\right)\left(|f|^{2}+\alpha\right), \\
E(s)=E(0)=\left|f^{\prime}(0)\right|^{2}+\frac{1}{4}\left(|f(0)|^{2}+\alpha\right)^{2}=\frac{\alpha^{2}}{4} .
\end{gathered}
$$

After a diagonalization the system (28) can be integrated from infinity by Picard iteration. In fact a similar result to Theorem 2 but for the ODE (25) can be obtained. Once this is done it is easy to conclude the proof of Theorem 2 in the case $a \neq 0$ due to some identities which do not exist if $a=0$. One of these identities is

$$
\left|T^{\prime}(s)\right|^{2}=-a T_{3}(s)-\alpha \quad \forall \alpha \in \mathbf{R},
$$

which also is fundamental in the proof of Theorem 3. If $a=0$ we are in the setting of Theorem 1, see [GRV].

\section{Planar Spirals}

Let us study the case of odd solutions of (15). Then $G(0)=0$ and by rotational invariance we can assume that $G^{\prime}(0)=\left(0,0, z_{3}\right),-1 \leq z_{3} \leq 1$.

Moreover, by the uniqueness of the solution we have that $A^{-}=\lim _{s \rightarrow-\infty} \frac{G(s)}{s}=$ $\lim _{s \rightarrow+\infty} \frac{G(s)}{s}=A^{+}$. Also the function $\beta\left(z_{3}\right)$

$$
\begin{aligned}
\beta:[-1,1] & \longrightarrow[-1,1] \\
z_{3} & \longrightarrow A_{3}^{+}
\end{aligned}
$$

is continuous and $\beta(1)=1$ and $\beta(-1)=-1$. Hence there must exist $\tilde{z}_{3}$ such that $\beta\left(\tilde{z}_{3}\right)=0$. In this case the asymptotic spiral is a planar logarithmical spiral and therefore

$$
X(s, t)=e^{\mathcal{A} / 2 \log t} \sqrt{t} G(s / \sqrt{t})
$$

will be asymptotically this planar logarithmic spiral for "all $t$ ". Recall that we are dealing with the binormal flow and therefore plane curves have to move perpendicularly to the plane where they are contained. This apparent contradiction is clarified from the asymptotics of the binormal vector given in Theorem 2 . 


\section{References}

[Be] R. Betchov, On the curvature and torsion of an isolated filament, Journal of Fluids Mechanics 22, (1965), 471.

[Bu] T. F. Buttke, A numerical study of superfluid turbulence in the Self-Induction Approximation, J. of Com. Phys. 76, (1988), 301-326.

[CFM] P. Constantin, C. Fefferman, A. Majda, Geometric constraints on potentially singular solutions for 3-D Euler equations, Comm. P.D.E. 21, (1988), 559571.

[DaR] L.S. Da Rios, On the motion of an unbounded fluid with a vortex filament of any shape, Rend. Circ. Mat. Palermo 22, (1906), 117.

[GRV] S. Gutiérrez, J. Rivas, L. Vega, Formation of singularities and self-similar vortex motion under the Localized Induction Approximation, To appear in Comm. P.D.E. .

[Ha] H. Hasimoto, A soliton on a vortex filament, Journal of Fluids Mechanics, 51, (1972), 477-485.

[LRT] M. Lakshmanan, T.H. W. Ruijgrok, C.J. Thompson, On the dynamics of a continuum spin system, Physica A 84, (1976), 577-590.

[Sa] P.G. Saffman, Vortex Dynamics, Cambridge Monographs on Mechanics and Applied Mathematics, Cambridge Univ. Press, New York, (1992).

Universidad del Pais Vasco, Spain

mtpvegol@lg.ehu.es 